\title{
Modulating microRNAs as Novel Therapeutic Targets in Cardiac Fibrosis
}

\author{
Zhongxiu Chen ${ }^{1 *}$, Yajiao $\mathrm{Li}^{*}$, Ke Dian${ }^{2}, \mathrm{Li} \mathrm{Rao}^{1 凶}$ \\ 1. Department of Cardiology, West China Hospital of Sichuan University, Chengdu, China; \\ 2. Department of Cardiovascular Surgery, West China Hospital of Sichuan University, Chengdu, China. \\ * These two authors contributed equally to this work. \\ $\bowtie$ Corresponding author: Li Rao. Fax: 86-28-85582944; E-mail: lrlz1989@163.com \\ (C) Ivyspring International Publisher. This is an open access article distributed under the terms of the Creative Commons Attribution (CC BY-NC) license \\ (https://creativecommons.org/licenses/by-nc/4.0/). See http://ivyspring.com/terms for full terms and conditions.
}

Received: 2017.01.21; Accepted: 2017.03.22; Published: 2017.06.01

\begin{abstract}
This commentary highlights the findings by Tao et al. (Theranostics 2016; 6: 2068-2083) that targeting miR-433 may be a potential therapeutic strategy for myocardial fibrosis and subsequently discusses the obstacles and prospects associated with the application of therapeutic microRNAs in anti-fibrosis treatment.
\end{abstract}

Key words: Cardiac fibrosis; microRNAs; Therapeutic targets.

\section{Introduction}

Myocardial fibrosis, a histopathologic hallmark of structural cardiac remodeling in most heart diseases such as myocardial infarction, cardiomyopathies, and heart failure, increase the risk of adverse cardiovascular events such as sudden cardiac death and ventricular dysfunction. Cardiac fibrosis is characterized by the disproportionate accumulation of various extracellular matrix components due to disrupted homeostasis between synthesis and degradation. Fibroblasts are known to play a critical role in the development of fibrosis. Despite its serious adverse effects, currently, there are no licensed treatments for cardiac fibrosis. microRNAs (miRs), small non-coding RNA molecules, are increasingly being considered not only as diagnostic biomarkers but also as promising novel therapeutic agents for cardiac fibrosis. Literature suggests that miR-29, miR-21, and miR-133 are the main miRs involved in the complex cardiac fibrosis process and that the altered expression of these miRs is associated with the fibrogenic cardiac phenotype $[1,2]$.

miR therapeutics involves the use of miR-mimics, antagomiRs, miR sponges, or direct repression of the miR transcript, and delivery of these
miRs by liposomes, viruses, or nanoparticles [3]. These miRs subsequently impact cardiac fibrotic processing as either suppressors of enhanced pro-fibrotic genes or mimics of reduced anti-fibrotic genes. Chemical modification of specific miRs, such as 2'-O-methylation, partial phosphorothioate backbones, and the attachment of a cholesterol moiety at the 3'-end, is the most advanced strategy, which can protect oligonucleotides against degradation in circulation and allow for cellular uptake. However, since these compounds need to be recognized by the miR processing system before exerting their function, high and repeated doses are currently required to ensure sufficient miR delivery. Additionally, low tissue specificity has limited the clinical applicability of this approach. Viral vectors and lipid nanoparticles have been developed for the successful systemic delivery of unmodified oligonucleotide miR-mimics. Helper molecules, such as fusogenic lipids, anionic lipids, and amphoteric liposomal complexes, have also been added to improve the efficiency of delivery and tissue specificity. Alternative approaches involving EDV particles, aptamer-conjugated miR-mimics, and miRNA regulators are used to a relatively lesser extent $[4,5]$. Strategies to use miRs as 
therapeutic targets in human diseases have rapidly progressed because of recent developments in nanotechnology and the application of molecular imaging in $\mathrm{miR}$ therapeutic evaluation [6]. Although these strategies have been tested primarily by targeting tumors or in liver diseases, the technical means used and the research progress achieved in these trials provide reference and basis for miR-therapeutic modulation in cardiac fibrosis. However, the degree of "off-target" effects of miRNA delivery also requires further investigation and is of greater concern compared to traditional treatment methods, since multiple similar pathways can be targeted by one miR.

A recently published article by Lichan Tao et al. [7] revealed that miR-433 plays a crucial role in the regulation of cardiac fibrosis and is a potential target for ameliorating cardiac fibrosis. According to their investigation, miR-433 levels were increased in the heart tissues of myocardium with fibrosis, and miR-433 inhibition exhibited a cardioprotective effect by targeting the AZIN1 and JNK1 genes through the TGF- $\beta 1$, ERK, and p38 kinase pathways. We appreciate their progressive work on anti-fibrotic therapies by regulating miRs; however, some issues should be considered when interpreting their results. First, cardiac remodeling is a complex process involving the coordinative interaction of numerous miRs, such as miR-24, miR-15, and miR-133 [2]. The effects of altering one of the RNA species on the unintended targets and pathological processes of the disease are not well-studied. Furthermore, numerous related mRNA genes and signaling networks under the control of the target miR would be affected; thus, the manipulation of a single miR can have a complex and profound impact on cellular phenotypes. Second, as discussed in the article, miR-433 is also present in other organs, such as the liver, kidney, and stomach; therefore, systemic delivery of miR-433 antagomiR may cause off-target effects in different tissues or cells. Unfortunately, the side-effects in other organs were not reported in this study. Although the authors attempted to attenuate these effects by using cardiotropic miR-433 sponge AAV9, AAV9 also showed affinity to skeletal muscle, alveolar, liver, and central nervous tissues. Therefore, the specificity of local delivery, in vivo stability, and binding affinity to the miR of interest should be considered before applying these findings in clinical settings.

Taken together, cardiac fibrosis is the proposed cause of arrhythmias and heart failure of most cardiomyopathies; however, no effective therapeutic strategies are currently available for this adverse remodeling. Increasing evidence has shown that miRs play a central role in cardiac fibrosis and that targeting specific miRs may be an attractive anti-fibrotic treatment option but several obstacles exist in the therapeutic use of miRs targeting the myocardium. There are no ongoing clinical trials for investigating the role of miRs in cardiac fibrotic changes. However, there has been remarkable progress in the development of desirable local delivery systems, such as miR-releasing biodegradable scaffolds, light-inducible antimiRs, and vesicle-encapsulated miRs $[8,9]$, which supports the promising potential of modulating miRs as an attractive therapeutic strategy for cardiac fibrosis.

\section{References}

1. Dong DL, Yang BF. Role of microRNAs in cardiac hypertrophy, myocardial fibrosis and heart failure. Acta Pharmaceutica Sinica B. 2011; 1: 1-7.

2. Wang X, Liu T, Zhao Z, Li G. Noncoding RNA in cardiac fibrosis. Int J Cardiol. 2015; 187: 365-368.

3. Abba ML, Patil N, Leupold JH, Moniuszko M, Utikal J, Niklinski J, et al. MicroRNAs as novel targets and tools in cancer therapy. Cancer Lett. 2017; 387: 84-94.

4. Krutzfeldt J. Strategies to use microRNAs as therapeutic targets. Best Pract Res Clin Endocrinol Metab. 2016; 30: 551-561.

5. Jin L, Zeng X, Liu M, Deng $Y$, He N. Current progress in gene delivery technology based on chemical methods and nano-carriers. Theranostics. 2014; 4: 240-255.

6. Sekar TV, Mohanram RK, Foygel K, Paulmurugan R. Therapeutic evaluation of microRNAs by molecular imaging. Theranostics. 2013; 3: 964-985.

7. Tao L, Bei Y, Chen P, Lei Z, Fu S, Zhang H, et al. Crucial Role of miR-433 in Regulating Cardiac Fibrosis. Theranostics. 2016; 6: 2068-2083.

8. Biglino G, Caputo M, Rajakaruna C, Angelini G, van Rooij E, Emanueli C. Modulating microRNAs in cardiac surgery patients: Novel therapeutic opportunities? Pharmacol Ther. 2016; 11: 004.

9. Samanta S, Balasubramanian S, Rajasingh S, Patel U, Dhanasekaran A, Dawn B.MicroRNA: A new therapeutic strategy for cardiovascular diseases. Trends Cardiovasc Med. 2016; 26: 407-419. 